\title{
Combining pervasive computing with activity recognition and learning
}

\author{
Patrice C. Roy ${ }^{1}$, Bruno Bouchard ${ }^{2}$, \\ Abdenour Bouzouane ${ }^{2}$ and Sylvain Giroux ${ }^{1}$ \\ ${ }^{1}$ DOMUS Laboratory, Université de Sherbrooke, (Canada) \\ ${ }^{2}$ LIAPA Laboratory, Université du Québec à Chicoutimi, (Canada)
}

\section{Introduction}

Today, networks, microprocessors, memory chips, smart sensors and actuators are faster, more powerful, cheaper and smaller than ever. Chips are all around, invading everyday objects. Wireless networks enable to easily connect them. Everyday objects can then propose innovative and unexpected interactions (Ullmer \& Ishii, 2000). Clothes will transport one's profile to reconfigure his environment according to his preferences (Abowd et al. 1997). Lamps will help people finding lost objects (Vergnes et al., 2005). Interactive portraits will reflect at distance the mood and health state of one's beloved relatives (Mynatt et al., 2001). This new technologically enhanced environment will enable finding novel solutions to help people in their everyday life, such as elders that suffer from cognitive deficit and have many difficulties to carry out their activities of daily living (Pigot et al., 2003). Most of these people wish to stay at home, where they feel comfortable and safe, as long as possible. The governments aim to help them for social reasons as well as economical ones. However, keeping cognitively impaired people at home involves many risks that are necessary to control. In order to do that, the physical and human environment must be specifically designed to compensate the cognitive impairments and the loss of autonomy (Ramos et al., 2008).

Combining pervasive computing with techniques from artificial intelligence (AI) greatly increases the acceptance of the pervasive assisted living and makes it more capable of providing a better quality of life in a non-intrusive way, where elderly people, with or without disabilities, could clearly benefit from this concept. From the computational perspective, there is a natural association between them. However, research addressing smart environments has in the past largely focused on network and hardware oriented solutions. AI-based techniques (planning and action theory, ontological and temporal reasoning, etc) that promote intelligent behaviour have not been examined to the same extent (Augusto \& Nugent, 2006), although notable exceptions can been found in the domain of activity recognition for healthcare. Prior work has been done to use sensors to recognize the execution status of particular types of activities, such as hand washing (Mihailidis et al., 2007), meal preparation (Barger et al., 2002), and movements around town (Liao et al., 2004). Additionally, several projects have attempted to do more general activity 
recognition, using radio frequency identification (RFID) tags attached to household objects and gloves (Philipose et al., 2004). At DOMUS and LIAPA labs, we investigate theory and praxis of activity recognition for cognitive assistance. Most theoretical and long term approaches are based on hierarchical task Markovian model (Bauchet \& Mayers, 2005; Pigot et al., 2008), Bayesian networks (Charniak \& Goldman, 1993), and lattice-based models (Bouchard et al., 2007) enhanced with probabilities (Roy et al., 2009) to recognize activities of daily living (ADLs) and to anticipate erroneous behaviours classified according to cognitive errors (Bouchard et al., 2008).

This chapter will investigate in details the challenging key issues that emerge from research in the field of activity recognition under the context of smart environment. We will clearly describe the specific functional needs inherent in cognitive assistance for effective activity recognition and then we will present the fundamental research works that try addressing this problem in such context. This chapter is more of a survey and an analysis of existing works that have been studied for potential integration into our laboratories, rather than a focused evaluation report. Our objective is to identify gaps in the capabilities of current techniques and to suggest the most productive lines of research to address this complex issue. The chapter is organized as follows. Section 2 presents the foundations that characterize the problem of activity recognition and position it in the context of smart environments. Section 3 draws a detailed portrait of the learning techniques for activity recognition that exist, and presents their forces and weakness in our specific context. Finally, we conclude this chapter with perspectives and future challenges of this fascinating issue.

\section{Activity recognition}

Activity recognition aims to recognize the actions and goals of one or more agents from a series of observations on the agent's actions and the environmental conditions (Kautz et al., 2003). Due to its many-faceted nature, different fields may refer to activity recognition as plan recognition. The problem of plan recognition has been an active research topic for a long time (Carberry, 2001) and still remains very challenging. The recognition problem corresponds to a fundamental question: how can we predict the behaviour of an observed or communicating agent, so that this prediction can be then used for task coordination, cooperation, assistance, etc.? From that definition, the expression activity recognition refers to the fact that one supposes the existence of an activity structure (i.e. a set of actions ordered in time) chosen at start by the acting entity (for instance a patient), which constitutes the result that the observing agent (for instance a smart environment) seeks to recognize. By referring to the literacy on the subject, one can realize that this vision of the problem is a historical heritage from the first expert systems, which was originally used to resolve planning issues (Waern \& Stenborg, 1995). The planning problem also constitutes a wellknown challenge in the AI community, which can be considered as the inverse of activity recognition (Russell \& Norvig, 2003). This difficulty consists, for an agent, to identify a sequence of actions (a plan) which, at the end of its execution, will allow the agent to achieve a pursued goal (Georgeff, 1987). For instance, an agent in a manufacturing environment can sense information from the environment and plan an action sequence in order to improve the manufacturing control system effectiveness (Merdan et al, 2006). Therefore, the problem of recognizing an activity can be seen as the inverse operation, in which another agent that does not know the planned objective has the task to identify the goal of the actor agent by 
inferring, from the observed actions, the possible on-going activities set. According to (Cohen et al., 1982) and (Geib \& Goldman, 2005), activity recognition can be characterized by the relationship that exists between the observed agent and its observer. This relationship allows dividing activity recognition into three different categories. Each of these recognition types makes a particular presumption about the existing relationship between the observing agent and the actor entity, which will guide the recognition process.

\subsection{Intended activity recognition}

In a context of intended recognition, one supposes that the agent explicitly knows that it is been observed and therefore, that it adapts deliberately its behaviour to perform its activity in a manner that will facilitate the recognition process of the observer (Kautz, 1991). Consequently, this form of recognition presumes a direct cooperative effort on behalf of the observed entity. This category of recognition was introduced by (Cohen et al., 1982). His goal was to position the activity recognition problem within the particular context of natural language processing, in which he worked at the time. Some researchers supposed, based on the cooperative assumption of this category, that the observer agent could directly ask the actor in case of uncertainty, in order to get clarifications on the on-going task (Lesh et al., 1998). From a pervasive assisted living viewpoint, it is unrealistic to position the problem of recognizing activities in a context of intended recognition. For instance, in the case of trying to assist an Alzheimer's patient in his every day tasks, it is clearly difficult to make any assumption about the capacity of the patient to sustain a cooperative effort by adapting his behaviour in order to positively influence the recognition process. Moreover, we cannot let the system to ask an observed patient for clarifications in case of uncertainty, because this action would result in an increased cognitive charge on behalf of the patient, which is unsuitable in a context of cognitive assistance.

\subsection{Adversarial activity recognition}

The second category, named adversarial recognition, has been introduced by (Geib \& Goldman, 2005) as a corollary of the first category, the intended recognition. In this type of recognition, rather than presuming that the observed agent will positively contribute to the recognition process, one supposes that the actor agent will likely attempt to spoil the process in a competitive gesture on its behalf (Mao \& Gratch, 2004). In other terms, the actor agent considers the observer agent as an enemy and therefore, it will voluntarily try to perform actions that are incoherent with its goals in order to lead the observer to infer false conclusions about its behaviour. This type of recognition suits better to a military context (Heinze et al., 1999) or to context where competitive agents are deployed inside video games to challenge a human player (Albrecht et al., 1998). However, this type of recognition does not fit well in pervasive assisted living context of cognitive assistance. Even if we need to take into account the fact that a cognitively impaired patient will likely perform activities in an erroneous way, as it is commonly the case in adversarial context to misguide the observer, these errors are not performed deliberately. They are rather the consequence of the patient's symptoms and not the result of a well-planned stratagem to compromise the recognition process. 


\subsection{Keyhole activity recognition}

The last category, named keyhole recognition, characterizes the assumption that an agent does not really know that it is been observed and thus, it will not attempt to influence the recognition process or to misguide the observer conclusions (Cohen et al., 1982). With this category, Cohen wanted to define a generic frame for activity recognition, in which one make a supposition of a neutral cooperative effort on behalf of the acting agent. Hence, Cohen made an analogy, from which the name of the category emanates, with a person being observed, inside a room, through the keyhole of the door, so that this person acts in the room naturally without worrying about being observed and without trying to help or compromise the on-going recognition process. We can position the activity recognition problem, from an assisted living point of view, in this category that suits better to the context of cognitive assistance, in which we certainly cannot presume the collaboration nor the deliberate nuisance of the patient (Bouchard, 2006).

The theory of keyhole plan recognition, on which we are working, tries to establish a formalization of this behavioural prediction. It is usually based on a probabilistic-logical inference engine for the construction of hypotheses about the possible plans, and on a matching process linking the observations with some plans included in a library or a model of activities related to the application domain. This library is an ontology of activity patterns that the observed agent can potentially carry out. At each observation of an action occurrence, the recognition agent tries to build hypotheses based on the knowledge described in this ontology. Since there can be many possible plans that can explain the observations, and thus the behaviour of the observed agent, the challenge is then to disambiguate these concurrent hypotheses. The researchers at DOMUS and LIAPA labs are exploring the following representation models to attack this issue.

\subsubsection{Lattice-Based Model}

The lattice plan recognition model tries to address the recognition issue by using lattice theory and Description Logics (DL) (Baader et al., 2007), which transforms the plan recognition problem into a classification issue. Description logics are a well-known family of knowledge representation formalisms that may be viewed as fragments of first-order logic. The main strength of DL is that they offer considerable expressive power going far beyond propositional logic, although reasoning is still decidable. The proposed model (Bouchard et al., 2007) provides an adequate basis to define algebraic tools used to formalize the inferential process of ADL recognition for Alzheimer's patients. To summarize, our approach consists of developing a model of minimal interpretation for a set of observed actions, by building a plan lattice structure as shown in the Figure 1. In this model, an intention schema characterizes the uncertainty related to the anticipated patient's behaviour. This schema corresponds to the lower bound of the lattice and is used to extract the anticipated incoherent plans, which are not pre-established in the knowledge base, that the patient may potentially carry out as a result of the symptoms of his disease. However, it is not sufficient to be able to disambiguate the relevant hypotheses. Therefore, the addition of a probabilistic quantification on the lattice structure (Roy et al., 2009) is an interesting and effective alternative, in the sense that it makes it possible to combine the symbolic approach for hypotheses construction with a probabilistic inferential process. The symbolic recognition agent filters the hypotheses by passing only a bounded lattice recognition space to the probabilistic inference engine, instead of considering the whole set of plans included 
in the ontology of the activities, as the classical probabilistic approaches usually do. The probabilistic quantification that we propose is based on samples of observation frequencies obtained at the end of a training period while the system learns the usual routines of the patient. This knowledge allows us to create a profile of the patient that offers a relevant basis to accurately estimate the probabilities of possible ongoing plans.

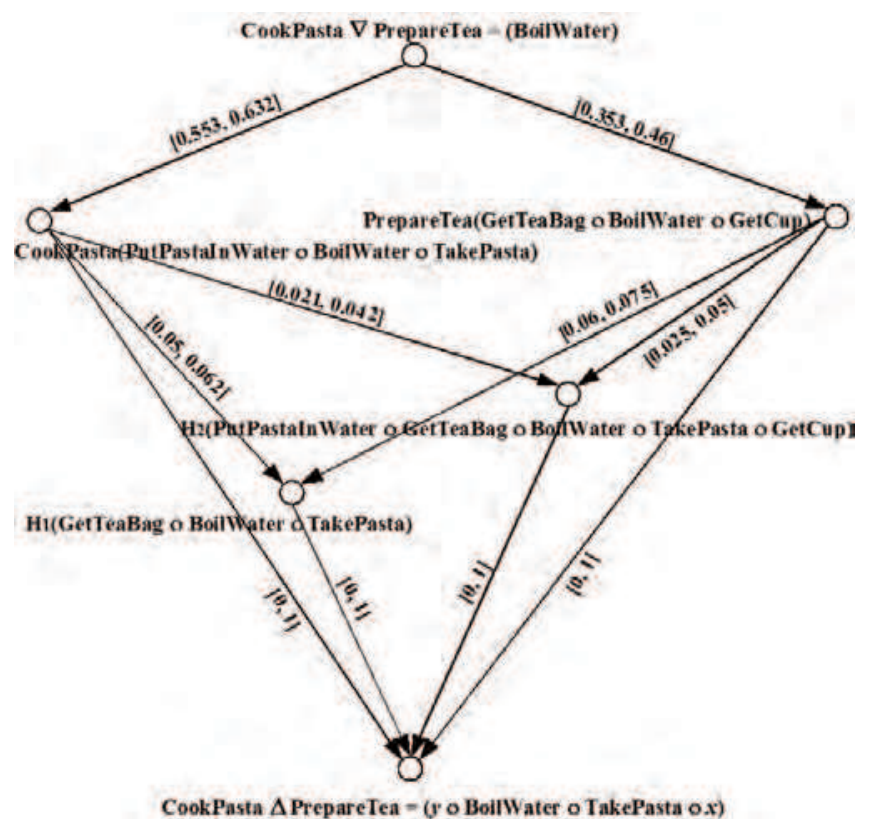

Fig. 1. A plan lattice structure that models two activities: cooking pasta and preparing tea.

This approach was implemented and tested in the DOMUS experimental smart home infrastructures, where we have simulated different scenarios based on 40 low-level actions and 10 activities of daily living. Each of these activities corresponds to a common kitchen task (cooking cake, cooking pasta, making tea, etc.) sharing several actions with some other activities, in order to create a realistic context where plans can be interleaved and can lead to many different kinds of planning errors (realization, initiation, sequence, completion, etc). The observation's frequencies of the erroneous and coherent behaviours are based on the frequencies described in the study of Giovannetti et al. (Giovannetti et al., 2002), done on 51 patients suffering from neurodegenerative diseases, which include the Alzheimer's disease. The results clearly show that the model recognizes all of the interleaved plans and realization type errors, and $70 \%$ of the sequence type errors. These results are promising, as all these recognized hypotheses were not pre-established in the knowledge base; they were dynamically generated in the recognition space, according to the initial identified possible plans set. However, our approach is limited by the fact that the first observed action is assumed to be correct (no errors) and coherent with the patient's goal. The problem is that in some scenarios that we simulated, the patient started by performing an action that he was only supposed to carry out in a later stage. This limitation explains the $30 \%$ of unpredicted sequence errors and also explains why our system has trouble predicting initiation errors. In 
another hand, we have also experimented the approach in concrete case by extending the system named COACH (Bouchard et al., 2008; Mihailidis et al., 2007), a cognitive aide for Alzheimer's patients that actively monitors an user attempting a handwashing task and offers assistance in the form of task guidance (e.g., prompts or reminders) when it is most appropriate. When an Alzheimer's patient is performing the handwashing activity, the system gets as observations a set of state variables obtained using cameras, such as the patient's hand location, the tap position (open or closed), etc., in order to determine the completion status of the task according to a previously handcrafted model. If the completion status of the task regresses or does not evolve for a certain period of time, the system will compute the best possible solution to achieve the task and will try to guide the person until the next activity step.

\subsubsection{Hierarchical task model}

Several investigators have highlighted the importance on representing hierarchically structured complex activities with dynamic probabilistic model. For instance, in Pigot et al. (Pigot et al., 2008) and Bauchet et al. (Bauchet \& Mayers, 2005), the recognition process is based on a model of activities where tasks are described using hierarchical structure as shown in the following figure.

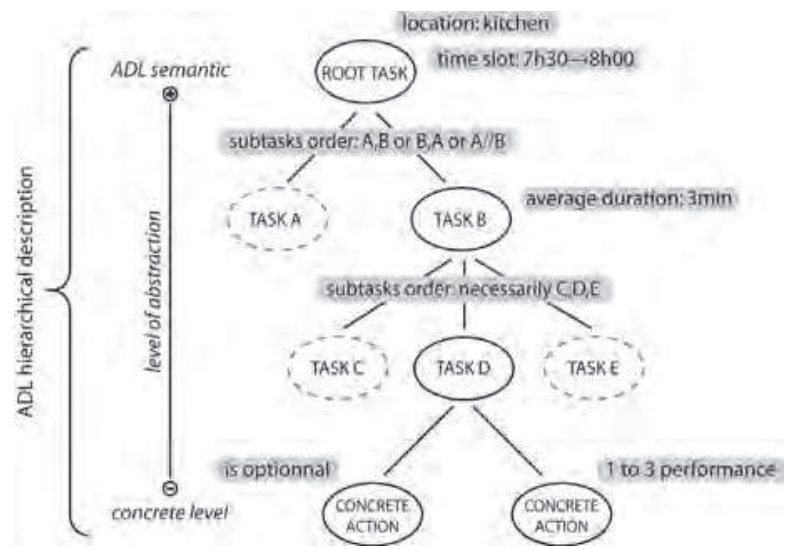

Fig. 2. ADL recognition process is based on a hierarchical model of activities.

The model includes two types of task's nodes: goal of the occupant and the method to complete it. Leaves are methods of terminal tasks, which mean an atomic way to realize a concrete goal. Similar approaches can be found in hierarchical task network planning. However, this hierarchical model does not consider the set of subtasks as a predefined sequence, since there are numerous ways to realize an activity for a given method. Instead of generating all plausible sequences, rules are defined to generalize, for a given method, the criteria of integration of subtasks: partial or total sequence, repetition and/or necessity constraint. Breaking those rules should be considered as an improper activity completion. To monitor the proper completion of activities, temporal information is introduced for tasks nodes. This deals with the average time needed to realize the task, and the time slot of completion. The validation of these constraints during task realization is done according to 
the Epitalk approach, a tutoring architecture used for generating advisor agents (Paquette et al., 1996). Each adviser manages a local model of the activity based on a hierarchical Markov model of the patient's habits by using an episodic memory. The activity is considered as an episode incorporating information on the method used for task completion, on right time slots, locations, sequences of subepisodes, frequencies of the observed activities, and so on. Hence, the adviser agent is both responsible to recognize a precise subtask and to provide assistance related to this task. The leaves of the model are connected to the IO events server and are fed by low-level events triggered by the sensors. A bottom-up traversal of the hierarchy aggregates information to provide for a larger view of what is going on.

The main characteristics of this model are that the plan recognition and the production of pieces of advice are combined into a single walk through the adviser tree. The principle is simple: each time a sensor triggers an event, it sends it to the corresponding terminal advisers. Then a bottom-up spreading is activated as follows: (i) each adviser (terminal or non-terminal) processes the information, either to issue local advice or to update a local model of the activity being observed, (ii) the adviser transmits to its direct father any information it considers relevant. This scheme is applied recursively for all advisers of the tree, terminal or non-terminal, until the root adviser is reached. Terminal advisers receive information directly from the host system, in particular sensors, whereas non-terminal advisers receive information from advisers below them in the hierarchy. This model, compared to previous works, allows a more effective description of ADLs for cognitive assistance. Despite the good results that has shown in real case assistance scenarios, the system appears to be somewhat limited owing to the fact that it is only able to monitor one specific ADL and the assistance agent react after the user error. This model constitutes the base component of the Archipel system (Bauchet et al., 2008) developed at DOMUS lab. Archipel is a context-oriented framework for cognitive assistance and has been applied to meal preparation. Its objectives are to promote ADLs completion for people with cognitive impairments, to foster their functional autonomy and their quality of life, and to exploit context-awareness and to use resources in the environment for assistance. A framework integrating four axis was implemented: knowledge representation, man-machine interfaces, ADL monitoring and ADL assistance.

\subsubsection{Bayesian networks and Markovian models}

In general, Bayesian networks are the principal technology used for performing activity recognition (Pollack, 2005). A typical approach is that taken in the Barista system (Patterson et al., 2007), which is a fine-grained ADL recognition system that uses object IDs to determine which activities are currently executed. It uses radiofrequency identification (RFID) tags on objects and two RFID gloves that the user wears in order to recognize activities in a smart home. The system is composed of a set of sensors (RFID tags and gloves) that detects object interactions, a probabilistic engine that infers activities with observations from the sensors, and a model creator that allows creating probabilistic models of activities from, for instance, written recipes. The activities are represented as sequences of activity stages. Each stage is composed of the objects involved, the probability of their involvement, and, optionally, a time to completion modelled as a Gaussian probability distribution. The activities are converted into Dynamic Bayesian Networks (DBN) by the probabilistic engine. By using the current sub-activity as a hidden variable and the set of objects seen and time elapsed as observed variables, the engine is able to probabilistically 
estimate the activities from sensor data. It was able to identify the specific on-going activity with recognition accuracy higher than $80 \%$, which is very impressive. This approach is able to identify the currently carried out ADL in a context where activities can be interleaved. However, this approach does not take into account the erroneous realization of activities, because the result of the activity recognition is the most plausible on-going ADLs.

Following the traces of works on Bayesian approaches, another branch of scientists (Boger et al., 2006; Patterson et al., 2005) recently proposed to define the activity recognition process by using Hidden Markov Models (HMM) formalism (Rabiner, 1990). This type of approach tries to represent the library of the observing agent with a set of discrete possible states. These states aim to characterize all the possible configurations of the smart environment, assuming that these configurations are not directly observable but can only be evaluated from inputs given by sensors. A stochastic model specifies and quantifies the dynamic that bound the transition between those states. The structure of the HMM is defined as a tuple $(S, O b s, A, B, \pi)$, where $S$ is the set of all possible states of the environment, Obs is the set of observable inputs given by sensors, $A$ (actions) is the probability transition matrix between states, $B$ is the probability matrix that links inputs from sensors with environment states, and $\pi$ defines the a priori probability of each state at the beginning of the recognition process, when there is no input.

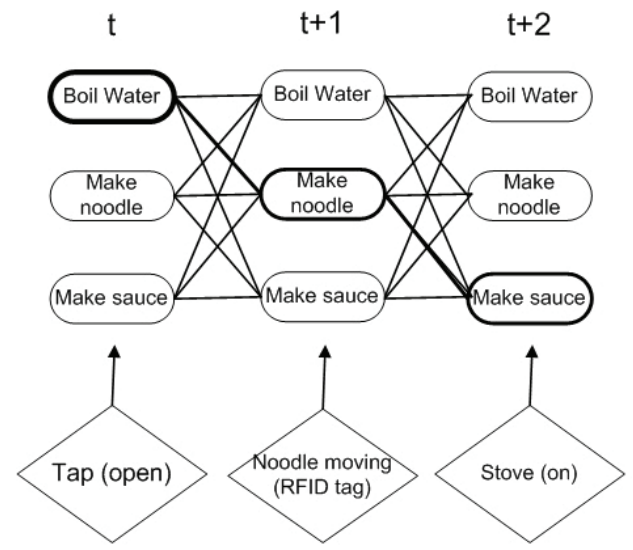

Fig. 3. Simple HMM for activity recognition in a smart environment.

Figure 3 shows an example of such HMM, where $S=\{$ "Boil water", "Make noodle", "Make sauce"\}, defining that the environment can be in three possible states: (1) the person is putting water to boil, (2) the person is making noodle, (3) the person is preparing sauce. In this example, we have three sensors, one on the tap, one on the noodle box and one on the stove. The probability of getting an input from the tap while being in the state "Boil water" could be, for instance, very high. Same thing with the noodle box sensor and "Make noodle", and the stove sensor and "Make sauce". Knowing the a priori probability of each state defined in $\pi$, and the probabilities matrix $A$ and $B$, the Viterbi algorithm (Forney, 1973) is used to estimate the most likely sequence of states that fit best with the sequence of observations. For instance, if the tap sensor came on at time $t$, the noodle box RFID tag indicates it is moving at time $t+1$, and the stove sensor came on at time $t+2$, the most likely inferred states 
sequence could be "Boil water", "Make noodle", "Make sauce". This sequence could then be linked with the corresponding activity, which could be here "Prepare Spaghetti".

At the end, it is clear that several limitations restraint the use of probabilistic models for recognizing activities in a context of ambient intelligence (AmI). The first problem is the complexity of calculations, notably with Bayesian's networks, at the time of the propagation in the network, mainly observed when the network's size is large. Most of these calculations are somewhat blind because the probabilities of all possible hypotheses are evaluated, even the one of irrelevant activities. Moreover, the estimation of the prior probabilities is a difficult and important task, because the probabilistic inference's precision depends on this estimation. In addition, the probabilistic distribution in the plan library must remain uniform. Therefore, when a new activity is added to the library, the probabilistic distribution must be re-evaluated in order to stay uniform. This constitutes a clear limitation in a context where one wishes to learn new behaviours coming from a patient. Furthermore, the result of the recognition consists of the most likely plan (activity) to be carried out, which constitutes a problem in a context where multiple activities can be performed in an interleaved or an erroneous way.

\subsection{Context-awareness and activity recognition}

When humans interact with each others, they are able to use implicit situational information, or context, in order to increase the efficiency of the interaction. In order to improve the effectiveness of computational services offered by smart home technologies, the context must be taken into account by the computing devices. Since the activity recognition process uses the current and previous situations of the environment to infer the behaviour of its occupant, contextual information must be retrieved from the environment sensors. There exist several definitions of the context concept. In (Schilit \& Theimer, 1994), a context is defined as the location, identities of nearby individuals and objects, and changes to those objects. In (Dey, 2001), a context is defined as any information that can be used in order to characterize the situation of an entity, which can be a person, place, or object that is considered relevant to the interaction between an user and an application, including the latter themselves. Systems that use or infer on contexts to provide contextual services are denoted to be context-awareness. One of the earlier definitions of a context-aware system is given in (Schilit \& Theimer, 1994), where a context-aware system is a system that adapts itself according to its location of use, the set of nearby objects and people, and the changes to those objects over time. In (Dey, 2001), a context-aware system uses context in order to provide relevant information and/or services to the user, where relevancy depends on the user's task.

There exist several models of context-aware systems. The approach of (Zhang et al., 2005) uses a layered model (context stack) to represent context-aware systems. The lowest level is the context acquisition layer, where context in raw data is acquired from the ubiquitous sensors. The second level is the context representation layer, where raw data are converted into an understandable and readable format according, for instance, to a context ontology. The next level, the context aggregation layer, aggregates contexts from distributed sensors in order to form a centralized context database, which allows to provide functionalities of a knowledge base and to store previous contexts. The fourth layer is the context interpretation layer, which uses reasoning/learning techniques to deduce implicit high-level context from explicit low-level context. Finally, the context utilization layer allows to context-aware 
services to use low and high-level contexts to adjust their behaviours. The model of (Ailisto et al., 2002) also subdivides context-aware systems into five layers. The lowest level is the physical layer, where sensors and objects in the environment produce output in a raw format. The next layer is the data layer, where objects produce processed data according to low-level sensors data. The third layer is the semantic layer, where data are transformed into a format where inference services can be applied. The fourth level, the inference layer, uses information from the semantic level and inference rules to make guesses on what the user is doing and what kind of services he might want. The last level, the application layer, uses the inferred context in order to plan a service task. In the activity recognition perspective, a context can be seen as raw data from the environment sensors in a particular situation or as an interpretation of the sensors data according to a knowledge representation language. Since the environment is partially observable, a context can be associated to a particular environment state or to a subset of possible environment states. The actions, activities or behaviours of the occupant inferred from the context can be seen as high-level contexts. By using hypotheses associated with those high-level contexts, a context-aware assistant application can plan and provide support tasks to the user if the situation requires it.

\section{Learning for activity recognition}

Another avenue of attack on the activity recognition problem is to apply learning techniques. These techniques seek to identify patterns from the observed actions set, in order to build a predictive model of the observed agent behaviour. A great number of these learning recognition approaches are based on probabilistic methods. For instance, Liao et al. (Liao et al., 2004) proposed a hierarchical Bayesian learning model for a person moving through a transportation network in an urban environment. This model aims to recognize and predict the future person's location and his transportation mode. This probabilistic learning technique is based on Rao-Blackwellised particle filters (Doucet et al., 2000), a variant of Bayes filters for estimating the state of a dynamic system. The main motivation for Liao's work is the development of a personal guidance system that will help cognitively impaired individuals to move safely and independently in their community. From this point of view, a significant contribution of these works is to provide a way to detect user errors and deviations from common routines by using a model-selection approach (Liao et al. 2004). This new feature, called by the authors novelty detection, is based on using two parallel stochastic models and on comparing the likelihood of the learned hierarchical model with a prior model. This method is interesting, as it gives a concrete means to the system to know when an assistance action would be required. The limitation of this modelselection approach is that, even if it recognizes the patient's new behaviour, it is still unable to determine if it corresponds to a coherent new routine or to an erroneous performance of a known plan. Moreover, this approach does not allow distinguishing between the different kinds of activity errors and is unable to evaluate the significance of the person's deviation. Wilson and Philipose (Wilson \& Philipose, 2005) have proposed a method for estimating how well an elder performs day-to-day activities and for suggesting to him some ways to enhance his performance. This method is based on a HMM and on the well-known Viterbi algorithm which is an algorithm used to find the most likely sequence of hidden states in a HMM, incorporating the notion of edit distance. When an elder person has failed in the completion of an activity, this method evaluates his performance, according to a set of 
correct examples performed and rated by a human professional, and finds the closest successful solution by computing the edit distance between the elder's performed action sequence and the learned examples. The strength of this approach is the capacity to evaluate precisely how bad the performance of the person's erroneous activity is, and also to propose an adapted correcting solution based on this specific erroneous performance. The main disadvantage of the method is that it cannot anticipate the possible abnormal behaviour of the patient, what is exactly what we try to do.

\subsection{Non-probabilistic Learning}

Regarding non-probabilistic learning techniques, Lent (Lent \& Laird, 2001) have worked on a general framework for learning-by observation systems based on inductive learning algorithms such as the C4.5 decision-tree learning (Quinlan, 1993). This approach tries to mimic human behaviour and models acquiring knowledge from observation as a machinelearning problem. The problem of this approach is how to manage the observations containing a great deal of noise. The observations of the human's behaviour consist of the sensor inputs, the human operator selections, and the actions performed to achieve these operators. Usually, these methods are effective in a deterministic environment, and if the agent's tasks were changed slightly, the decision trees would have to be relearned from a new set of observations. Tambe (Tambe et al., 2000) has conducted some experiments, using a C4.5 decision-tree approach, on building an agent for helping user scheduling meetings based on observation learning of transfer decision making between a human and his agent. This agent fails to address the adjustable autonomy challenge in a team context, due to the fact that in this kind of logically based learning approaches, the generalization of learned rules might lead to inferring inconsistent behaviour (in this case, the agent incorrectly cancelled a meeting because it over-generalized from training examples).

\subsection{Applying Learning Techniques to ADL Recognition}

Over the last few years, a great amount of work has used learning approaches, mostly probabilistic ones, to address the problem of ADL recognition for cognitively impaired patients. In their assisted cognition project (Patterson et al., 2002) have developed Activity Compass, a cognitive aide for early-stage Alzheimer's patients. This system is based on the learning recognition model described in (Liao et al., 2004). In his work, Patterson directly addresses the issue of incoherent behaviour recognition and proposes to identify the Alzheimer's patient plans that are incomplete or improperly formed, by matching them to the closest learned pattern. Cook's team in their MavHome project (Singla et al., 2008) argues that if one can successfully recognize ADL initiation and completion in the context where the activity is incomplete or the resident may switching between tasks, then one can use the technologies to perform automatic assessment of an individual's well being and provide the foundation for reminder-based interventions. They designed algorithms that probabilistically identify the activity while it is performed, as well as identify steps that are missing. They employ a HMM augmented with temporal information to automatically learn the initiation and completion of ADLs. They demonstrate by showing an overall accuracy of $88.63 \%$ that this temporal information improves activity recognition performance on real-world task as performed in their smart home testbeds. Once again, theses approaches cannot distinguish the different types of patient's deviations and needs a 
long training period to be efficient. Moreover, the patient's habits may change from time to time, according to new experiences, the hour of the day, his physical and psychological condition, etc. Therefore, the patient's routines must be constantly re-learned and an adaptation period is required by the system.

\section{Ongoing and future work}

The new development towards pervasive assisted living will stimulate the research in many fields of artificial intelligence, such as multi-agent approach as development paradigm for this open and hardly dynamic environment. Since forty years, artificial intelligence has not ceased to being used on a large scale through expert system applications, web search agent, etc. If the internet devoted the advent of the conventional planetary networks, the next evolution, that will support the development of the artificial intelligence, relates to new challenging issues concerning how a network of agents will be deployed within our natural living environment, and how each of these artificial agents, in the sense of multi-agent systems, will be represented according to the following ambient capacities: (i) ubiquity which means that the agent must be able to interact with an embarqued heterogeneous electronic devices by using pervasive computing, (ii) context-awareness based on ontological reasoning to detect the localization and the implication of objects and inhabitants in daily activities, (iii) natural interaction for communicating intuitively with occupant through a personalized multimodal interface, and finally (iv) intelligence based on activity recognition and machine learning in order to predict the behaviour of the inhabitant in the case of assisted living allowing cognitive assistance and as well as stimulation for avoiding the rejection of such new technology. Hence, the question concerns the integration of these four characteristics within any objects of everyday life. For instance, if the door of the refrigerator is open, the associated ambient agent must be able to have an idea on the behaviour of the person, such as this opening is under the context of meal preparation while it communicates in an opportunistic way with other objects of the habitat, for example, the cooker's ambient agent. The stimulation for closing the door because of memory loss can be done through an intuitive interaction (game) between the refrigerator's ambient agent and the occupant with disabilities, explaining the concept of the door closed. This new concept of ambient agent will ineluctably impose a capital evolution, for instance, in the assisted living. As a contribution, this chapter investigated in details the challenging key issues related to the development of ambient agents in smart environment, under the context of activity recognition, by defining the notion of activity recognition in the context of AmI, and by presenting a survey and an analysis of existing works on activity recognition that have been studied for potential integration into our respective laboratories. This chapter allows one to identify gaps in the capabilities of current techniques and to give hints on the most productive lines of research to address this complex issue. We raised numerous research challenges that need to be addressed for understanding the domain and enabling ambient multi-agent recognition systems for cognitive assistance to operate effectively. 


\section{References}

Abowd, G.D.; Dey, A.K.; Orr, R. \& Brotherton J.A. (1997). Context-awareness in wearable and ubiquitous computing, Proceedings of the 1st International Symposium on Wearable Computing (ISWC '97), pp. 179-180, Cambridge, USA, October 13-14, 1997.

Ailisto, H.; Alahuhta, P.; Haataja, V.; Kylloenen, V. \& Lindholm, M. (2002). Structuring context aware applications: Five-layer model and example case, Proceedings of the Workshop on Concepts and Models for Ubiquitous Computing, pp.1-5, Goteborg, Sweden.

Albrecht, D.W.; Zukerman, D.W. \& Nicholson, A.E. (1998). Bayesian Models for Keyhole Plan Recognition in an Adventure Game. User Modeling and User-Adapted Interaction, Vol.8, No.1-2, pp. 5-47.

Augusto, J.C. \& Nugent, C.D. (2006). Designing Smart Homes: The Role of Artificial Intelligence, State of the Art Survey, vol. LNAI 4008, Springer-Verlag, 2006.

Baader, F.; Calvanese, D.; McGuinness, D.L.; Nardi, D. \& Patel-Schneider, P.F. (2007). The Description Logic Handbook: Theory, Implementation, and Applications. Second Edition, Cambridge University Press.

Barger, T.; Alwan, M.; Kell, S.; Turner, B.; Wood, S. \& Naidu, A. (2002). Objective remote assessment of activities of daily living: Analysis of meal preparation patterns, Medical Automation Research Center, University of Virginia Health System, Charlottesville.

Bauchet, J.; Giroux, S.; Pigot, H.; Lussier-Desrochers, D. \& Lachapelle Y. (2008). Pervasive Assistance in Smart Homes For People with Intellectual Disabilities: A Case Study on Meal Preparation. IJARM, Vol. 9, pp. 53-65.

Bauchet, J. \& Mayers, A. (2005). Modelisation of ADLs in its Environment for Cognitive Assistance, Proc. of the 3rd International Conference on Smart homes and health Telematic, ICOST'05, pp.3-10, Sherbrooke, Canada.

Boger, J.; Hoey, J.; Poupart, P.; Boutilier, C.; Fernie, G. \& Mihailidis, A. (2006). A Planning System Based on Markov Decision Processes to Guide People with Dementia Through Activities of Daily Living. IEEE Transactions on Information Technology in BioMedicine, Vol.10, No.2, pp.323-333.

Bouchard, B. (2006). Un modèle de reconnaissance de plan pour les personnes atteintes de la maladie d'Alzheimer basé sur la théorie des treillis et sur un modèle d'action en logique de description, Ph.D. Thesis, University of Sherbrooke, Canada, 268 pages.

Bouchard, B.; Bouzouane, A. \& Giroux, S. (2007). A Keyhole Plan Recognition Model for Alzheimer's Patients: First Results, Applied Artificial Intelligence: An International Journal, Vol.22, No. 7, pp. 623-658.

Bouchard, B.; Roy, P.C.; Bouzouane, A.; Giroux, S. \& Mihailidis, A. (2008). Towards an extension of the $\mathrm{COACH}$ task guidance system: Activity recognition of alzheimer's patients. ECAI'08 3rd Workshop on Artificial Intelligence Techniques for Ambient Intelligence (AITAmI'08), pp. 16-20, Patras, Grece.

Carberry, S., Techniques for plan recognition, (2001). User Modeling and User-Adapted Interaction, Vol.11, No.1-2, pp. 31-48.

Charniak, E. \& Goldman, R.P. (1993). A Bayesian model of plan recognition. Artificial Intelligence, Vol.64, No.1, pp.53-79. 
Cohen, P.R.; Perrault, C.R. \& Allen, J.F. (1982). Beyong question answering, in Strategies for Natural Language Processing, Lehnert, W.G. \& Ringle, M.H. eds., Lawrence Erlbaum Associates, Hillsdale, NJ.

Dey, A.K. (2001). Understanding and Using Context. Personal and Ubiquitous Computing, Vol.5, No.1, pp.4-7.

Doucet, A.; Freitas, N.; Murphy, K. \& Russell, S. (2000). Rao-Blackwellised particle filtering for dynamic Bayesian networks. Annual Conference on Uncertainty in Artificial Intelligence (UAI'00), pp. 176-183.

Forney, G.D. (1973). The Viterbi algorithm. Proc. of IEEE, Vol.61, No.3, pp. 268-278.

Heinze, C.; Goss, S.; Lloyd, I. \& Pearce, A. (1999). Plan Recognition in Military Simulation : Incorporating Machine Learning with Intelligent Agents. Proc. of IJCAI-99 Workshop on Team Behaviour and Plan Recognition, pp. 53-64.

Geib, C. \& Goldman, R. (2005). Partial Observability and Probabilistic Plan/Goal Recognition, Proc. of the IJCAI-05 workshop on Modeling Others from Observations (MOO-05), Int. Joint Conference on Artificial Intelligence, pp.1-6.

Georgeff, M.P. (1987). Planning, Annual Review of Computer Science, Vol.2, pp. 359-400, Annual Reviews, Palo Alto, California.

Giovannetti, T.; Libon, D.J.; Buxbaum, L.J. \& Schwartz, M.F. (2002). Naturalistic action impairments in dementia, Neuropsychologia, Vol.40, No.8, pp.1220-1232.

Kautz, H. (1991). A Formal Theory of Plan Recognition and its Implementation, in: Reasoning About Plans, Allen, J.; Pelavin, R. \& Tenenberg, J. eds., pp.69-125, Morgan Kaufmann, San Mateo, CA.

Kautz, H.; Etzioni, O.; Fox, D. \& Weld, D. (2003). Foundations of assisted cognition systems. Tech. Rep. CSE-02-AC-01, University of Washington.

Lent, M. and Laird, J.E. (2001). Learning Procedural Knowledge Through Observation, Proceedings of the 1st international conference on Knowledge capture, pp.179-186, Victoria, British Columbia, Canada.

Lesh, N.; Rich, C. \& Sidner, C.L. (1998). Using plan recognition in Human-Computer Collaboration, TR-98-23, Mitsubishi Electric Research Lab (MERL).

Liao, L.; Kautz, H. \& Fox, D. (2004). Learning and inferring transportation routines. Proceedings of the 19th National Conference on Artificial Intelligence (AAAI), pp.348-353, San Jose, California.

Merdan, M.; Kordic, V.; Zoitl, A. \& Lazinica, A. (2006) Knowledge-based Multi-agent Architecture, Proc. of the International Conference on Computational Intelligence for Modelling Control and Automation and International Conference on Intelligent Agents, Web Technologies and Internet Commerce (CIMCA-IAWTIC'06), pp.1-6, Sydney, Australia.

Mao, W. \& Gratch, J. (2004). Decision-Theoric Approach to Plan Recognition. Technical Report ICT-TR-01-2004, Institute for Creative Technologies (ICT).

Mihailidis, A.; Boger, J.; Canido, M. \& Hoey, J. (2007). The use of an intelligent prompting system for people with dementia, Interactions, Vol. 14, pp.34-37.

Mynatt, E.D.; Rowan, J.; Craighill, S. \& Jacobs, A. (2001). Digital family portraits: Supporting peace of mind for extended family members. Proc. of the ACM Conference on Human Factors in Computing Systems (CHI 2001), pp. 333-340, Seattle, Washington, ACM Press. 
Paquette, G.; Pachet, F.; Giroux, S. \& Girard, J. (1996). Epitalk, generating advisor agents for existing information systems. Artificial Intelligence in Education, Vol.7, No.3-4, pp.349-379.

Patterson, D.J.; Etzioni, O. \& Kautz, H. (2002). The Activity Compass. Proc. of the 1st Int. Workshop on Ubiquitous Computing for Cognitive Aids, pp.1-3, Gothenberg, Sweden.

Patterson, D.J.; Fox, D.; Kautz, H.A. \& Philipose, M. (2005). Fine-Grained Activity Recognition by Aggregating Abstract Object Usage, Proc. of the IEEE International Symposium on Wearable Computers, pp.1-8, Osaka, Japan.

Patterson, D.J.; Kautz, H.A. ; Fox, D. \& Liao L. (2007) Pervasive computing in the home and community. In: Pervasive Computing in Healthcare, Bardram, J.E.; Mihailidis, A. \& Wan, D. (Eds.), pp.79-103, CRC Press, Boca Raton, FL.

Philipose, M.; Fishkin, K.P.; Perkowitz, M.; Patterson, D.J.; Hahnel, D.; Fox, D. \& Kautz H., (2004). Inferring Activities from Interactions with Objects, IEEE Pervasive Computing, Vol.3, No.4, pp. 50-56.

Pigot, H.; Lussier-Desrochers, D.; Bauchet, J.; Lachapelle, Y. \& Giroux, S. (2008). A Smart Home to Assist in Recipe Completion. In: Technology and Aging, Assistive Technology Research Series, vol. 21, Mihailidis, A.; Kautz, H.; Boger, J. \& Normie, L., Eds., pp.3542, IOS Press, Amsterdam.

Pigot, H.; Mayers, A. \& Giroux, S. (2003). The intelligent habitat and everyday life activity support, Proc. Int. Conf. in Simulations in Biomedicine, pp.507-516, Slovenia.

Pollack, M.E. (2005). Intelligent Technology for an Aging Population: The Use of AI to Assist Elders with Cognitive Impairment, AI Magazine, Vol.26, No.2, pp.9-24.

Quinlan, J.R. (1993). C4.5: Programs For Machine Learning, Morgan Kaufmann, San Mateo, CA.

Ramos, C.; Augusto, J.C. \& Shapiro, D. (2008). Ambient Intelligence: the Next Step for Artificial Intelligence, IEEE Intelligent Systems, Vol. 23, No. 2, pp.5-18.

Rabiner, L.R. (1990). A tutorial on hidden Markov models and selected applications in speech recognition. IEEE Vol.77, No.2, pp.267-296.

Roy, P.C.; Bouchard, B.; Bouzouane, A. \& Giroux, S., (2009). A hybrid plan recognition model for Alzheimer's patients: interleaved-erroneous dilemma, Web Intelligence and Agent Systems (WIAS): An Int. Journal, Vol.7, No.4, pp.1-23, (to appear).

Russell, S. \& Norvig, P. (2003). Artificial Intelligence: A Modern Approach, $2^{\text {nd }}$ edition. PrenticeHall, Englewood Cliffs, NJ.

Schilit, B.N. \& Theimer, M.M. (1994). Disseminating active map information to mobile hosts. IEEE Network, Vol.8, No.5, pp.22-32.

Singla, G.; Cook, D. \& Schmitter-Edgecombe, M. (2008). Incorporating temporal reasoning into activity recognition for smart home residents. Proceedings of the AAAI Workshop on Spatial and Temporal Reasoning, pp.1-9.

Tambe, M.; Pynadath, D.V.; Chauvat, N.; Das, A. \& Kaminka, G.A. (2000). Adaptive agent integration architectures for heterogeneous team members. International Conference on MultiAgent Systems, pp. 301-308.

Ullmer, B. \& Ishii H. (2000). Emerging frameworks for tangible user interfaces. IBM Systems Journal, Vol.39, No.3-4, pp.1-15.

Vergnes, D.; Giroux, S. \& Chamberland-Tremblay, D. (2005). Interactive assistant for activities of daily living. 3rd International Conference on Smart Homes and Health Telematics, ICOST 2005, pp.229-236, July 4-6, Sherbrooke, Canada. 
Waern, A. \& Stenborg, O. (1995). Recognizing the plans of a replanning user. IJCAI-95 Workshop on The Next Generation of Plan Recognition Systems, pp.113-118, Montréal, Canada.

Wilson, D. \& Philipose, M. (2005). Maximum a posteriori path estimation with input trace perturbation: algorithms and application to credible rating of human routines. Nineteenth International Joint Conference on Artificial Intelligence (IJCAI'05), pp.895901, Edinburgh, Scotland, UK.

Zhang, D.; Gu, T. \& Wang, X. (2005). Enabling Context-aware Smart Home with Semantic Technology. International Journal of Human-friendly Welfare Robotic Systems, Vol.6, No.4, pp1-14. 


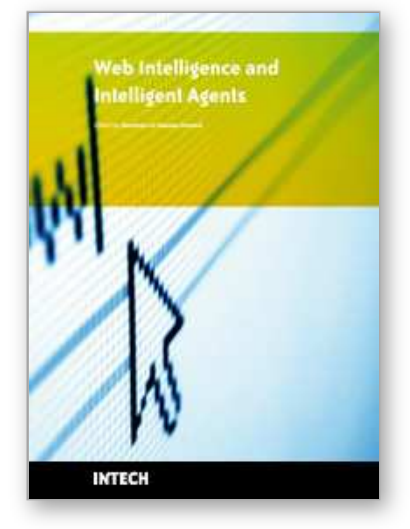

\section{Web Intelligence and Intelligent Agents \\ Edited by Zeeshan-UI-Hassan Usmani}

ISBN 978-953-7619-85-5

Hard cover, 486 pages

Publisher InTech

Published online 01, March, 2010

Published in print edition March, 2010

This book presents a unique and diversified collection of research work ranging from controlling the activities in virtual world to optimization of productivity in games, from collaborative recommendations to populate an open computational environment with autonomous hypothetical reasoning, and from dynamic health portal to measuring information quality, correctness, and readability from the web.

\section{How to reference}

In order to correctly reference this scholarly work, feel free to copy and paste the following:

Patrice C. Roy, Bruno Bouchard, Abdenour Bouzouane and Sylvain Giroux (2010). Combining Pervasive Computing with Activity Recognition and Learning, Web Intelligence and Intelligent Agents, Zeeshan-UIHassan Usmani (Ed.), ISBN: 978-953-7619-85-5, InTech, Available from:

http://www.intechopen.com/books/web-intelligence-and-intelligent-agents/combining-pervasive-computingwith-activity-recognition-and-learning

\section{INTECH}

open science | open minds

\section{InTech Europe}

University Campus STeP Ri

Slavka Krautzeka 83/A

51000 Rijeka, Croatia

Phone: +385 (51) 770447

Fax: +385 (51) 686166

www.intechopen.com

\section{InTech China}

Unit 405, Office Block, Hotel Equatorial Shanghai

No.65, Yan An Road (West), Shanghai, 200040, China

中国上海市延安西路65号上海国际贵都大饭店办公楼 405 单元

Phone: +86-21-62489820

Fax: $+86-21-62489821$ 
(C) 2010 The Author(s). Licensee IntechOpen. This chapter is distributed under the terms of the Creative Commons Attribution-NonCommercialShareAlike-3.0 License, which permits use, distribution and reproduction for non-commercial purposes, provided the original is properly cited and derivative works building on this content are distributed under the same license. 